\title{
SIMULATING LARGE AREA, HIGH INTENSITY AMO ILLUMINATION - TEST RESULTS FROM BEPICOLOMBO AND SOLAR ORBITER QUALIFICATION
}

\author{
C. Oberhüttinger ${ }^{(1)}$, H. Nesswetter ${ }^{(1)}$, D. Quabis ${ }^{(1)}$, C. G. Zimmermann ${ }^{(1)}$ \\ (I)Airbus Defence and Space, 81663 Munich, Germany,Email: carola.oberhuettinger@airbus.com
}

\begin{abstract}
Two ESA high temperature missions, BepiColombo and Solar Orbiter, are planned to start in 2018. Severe environmental conditions with sun intensities up to 10.6 resp. 12.8 solar constants will be encountered during both missions.

Different tests have been performed to qualify the main component for the solar array, the solar cell. The key qualification test for these high temperature, high intensity missions is a combined test which exposes a large number of cells simultaneously to the complete AM0 spectrum at the required irradiance and temperature.

During the last European Space Power Conference in 2014, the development and performance of the appropriate test setup in the VTC1.5 chamber located at ESTEC was presented. In the meanwhile, the BepiColombo 4000 hour qualification test is successfully finished. This paper summarizes the results of the qualification test and highlights the main findings.

Specifically, the following topics will be treated. Different methods like electrical performance, thermooptical and external quantum efficiency measurements have been used to characterize the behaviour of the solar cells after illumination under these conditions. A special focus has been put on the electrical performance. A comparison to other solar cell qualification tests under solely UV radiation has been undertaken. The results have also been compared to a theoretical model. However, the paper will not cover only characterization results but will also give some insight in challenges experienced during the test execution itself. Deviating from other solar cell qualification tests, a representatively equipped photovoltaic assembly on carbon fibre reinforced cyanate has also been included. On these coupon segments, solar cell assemblies connected to shunt diodes and placed next to optical surface reflectors have been exposed to AM0 illumination to qualify the solar cells including their surroundings which therefore covers also contamination effects. Last but not least, first results from the Solar Orbiter qualification are presented. This test with additional 1000 hours and increased intensity has been completed recently.
\end{abstract}

\section{INTRODUCTION}

Two ESA high temperature missions, BepiColombo (BC) and Solar Orbiter (SolO), are planned to start in
2018. The severe environmental conditions with sun intensities up to 10.6 resp. 12.8 solar constants (SCs) results in predicted solar cell operating temperatures up to $205^{\circ} \mathrm{C}$. A specific cell design was therefore developed for these missions [1,2]. Different tests have been performed to qualify the solar array according to the ECSS standards [3]. For one of the main components, the solar cell, the key qualification test for these high temperature, high intensity missions was a combined test which exposes a large number of cells simultaneously to the complete AM0 spectrum at the required irradiance and temperature. In the last European Space Power Conference (ESPC), the development and performance of the corresponding test setup was presented [4]. This paper now covers the results of the completed qualification test.

\section{QUALIFICATION TEST DESCRIPTION}

The BepiColombo AM0 Long Duration Illumination Qualification Test is a $4000 \mathrm{~h}$ test which was conducted from November 2013 to September 2015 in the VTC1.5 chamber located at ESTEC. The illumination intensity was set to $10 \mathrm{SCs}+/-2.5 \mathrm{SCs}$ AM0. Solar cell test temperature was $190^{\circ} \mathrm{C}$ resp. $205^{\circ} \mathrm{C}(600 \mathrm{~h})$. Both solar cell assemblies (SCAs) and representatively equipped photovoltaic assembly (PVA) on carbon fibre facesheets have been investigated. They were individually connected to an external shunt resistor to operate them close to the maximum power point. The samples have been mounted on a specifically developed mechanical ground support equipment (MGSE) which provides the possibility to test the samples in the light beam under an angle of $70^{\circ}$.

For monitoring reasons, different additional equipment has been used. On the one hand, molecular organic contamination control samples have been used to determine quantitatively and qualitatively the occurring contamination. On the other hand, an infrared (IR) camera was placed inside the chamber to examine the arising solar cell temperatures. This camera was providing live pictures during the complete test duration and was accessible via internet. Furthermore, thermocouples on some witness SCAs as well as the current and voltage of the individual SCAs and coupon segments under qualification were continuously recorded and checked by remote access.

Due to lamp restrictions, the complete test duration had to be split into sub-cycles with envisaged duration of about 400 hours for each cycle. The test breaks have 
been used for detailed solar cell characterizations at ambient conditions.

As the Solar Orbiter mission submits the solar array to even higher sun intensities, the SolO representative solar cells were tested for further 1000 hours at the required increased intensity of $10.5 \mathrm{SCs}+/-2.3 \mathrm{SCs}$ and $190^{\circ} \mathrm{C}$ using the same setup. This additional test was performed from May to August 2016.

\section{BEPICOLOMBO SOLAR CELL QUALIFICATION TEST RESULTS}

As qualification samples, $1640 \times 40 \mathrm{~mm}^{2}$ solar cells and $1780 \times 40 \mathrm{~mm}^{2}$ solar cells with the specific design for high temperature, high intensity missions and equipped with interconnectors and cover glasses have been placed inside the VTC1.5 chamber for the test on SCA level. These SCAs have been bonded to aluminium holder plates for handling reasons. The following inspections have been used to characterize the samples: visual inspection, electroluminescence measurements (ELM), electrical performance measurements, dark IV, thermooptical properties and external quantum efficiency (EQE). In the following subsections, the main findings of certain measurement techniques are summarized.

\subsection{Live Data Analysis of the Cell Parameters}

The individual cell currents and voltages were recorded with a sampling rate of $300 \mathrm{~s}$. Due to the lamp movement and degradation, which is described in section 5.1, the intensity on the samples was varying. This also lead to a slightly changing temperature on the SCAs and because of the temperature/intensity dependence of the cell parameters the current and voltage values were also varying. These effects overweigh the minor degradation of the cells which is presented below.

However, with the continuous live data it is possible to detect major changes in cell performance. This was used to determine the occurrence time for a cell crack. For the corresponding cell a crack was detected during ELM inspection after test cycle 4 . Fig. 1 shows for the test cycles $2-5$ the voltage of that cell related to the neighboring cells, to exclude lamp variations as good as possible. The moving average (black curve) was calculated to reduce the remaining fluctuations even more. During cycles $2-4$ the affected cell had a voltage of about $98 \%$ of the cells next to it and in cycle 5 it was only $96 \%$. A cell crack reduces the maximum power point voltage because of increased surface recombination, what confirms that the crack occurred between lamp switch-off for cycle 4 and the ELM inspection. It could therefore be concluded that the cell crack did not occur during the exposure test itself.

\subsection{Electrical Performance}

The main focus of the test was on the evolution of the

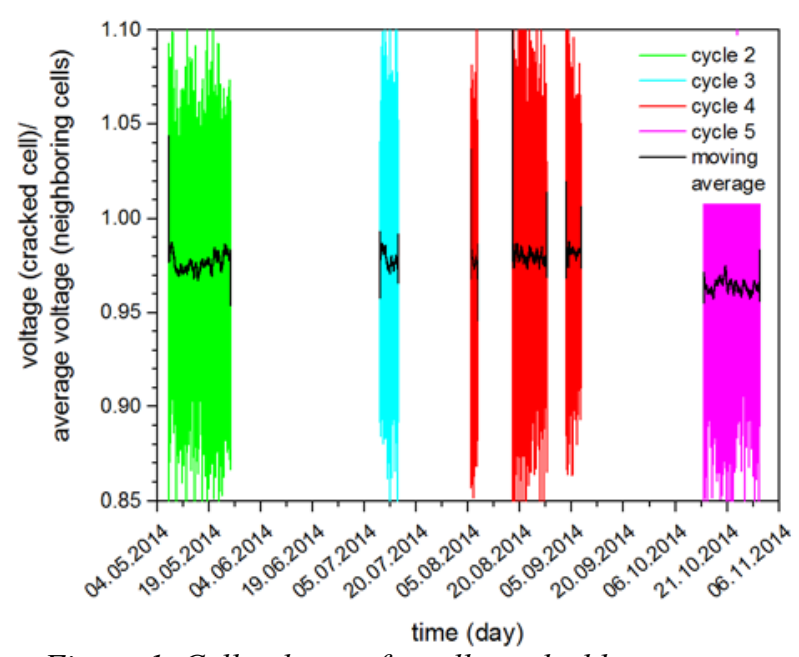

Figure 1. Cell voltage of a cell cracked between test cycle 4 and 5 related to the neighboring cells.

electrical characteristics of the solar cells at the different test steps. In average there is no difference for the 40x40 $\mathrm{mm}^{2}$ and $80 \times 40 \mathrm{~mm}^{2}$ solar cells. Therefore, Tab. 1 shows the average degradation in maximum power $\left(P_{\max }\right)$, voltage $V_{p \max }$ at $P_{\max }$, current $I_{p \max }$ at $P_{\max }$, short circuit current $\left(I_{s c}\right)$, open circuit voltage $\left(V_{o c}\right)$, and fill factor FF of all SCAs after $4000 \mathrm{~h}$ test.

\begin{tabular}{|c|c|c|c|c|c|}
\hline $\begin{array}{c}\Delta P_{\max } \\
(\%)\end{array}$ & $\begin{array}{c}\Delta V_{p \max } \\
(\%)\end{array}$ & $\begin{array}{c}\Delta I_{p \max } \\
(\%)\end{array}$ & $\begin{array}{c}\Delta I_{s c} \\
(\%)\end{array}$ & $\begin{array}{c}\Delta V_{o c} \\
(\%)\end{array}$ & $\begin{array}{c}\Delta F F \\
(\%)\end{array}$ \\
\hline-3.0 & -0.2 & -2.8 & -2.3 & -0.3 & -0.4 \\
\hline
\end{tabular}

Table 1. Average degradation of all SCAs after $4000 \mathrm{~h}$ test duration.

The main degradation is related to the current parameters and consequently a bit more pronounced to $P_{\max }$. This gives a clear indication that the degradation is mainly caused by transmission losses, i.e. cover glass adhesive darkening and contamination.

In Fig. 2, the average degradation in $I_{s c}$ of all samples is plotted over the test hours. It was of special interest if the observed behaviour is comparable to results obtained in further solar cell qualification tests under solely UV illumination. Therefore, test data from corresponding UV tests for BepiColombo have been included as well. In theory, the UV degradation $D$ over time $t$ of photovoltaic modules can be calculated according to Eq. (1) [5]:

$$
D(t)=1-n \cdot \ln (1+a \cdot t)
$$

with parameters $n$ and $a$ derived from a fit to the UV test results. The theoretical degradation describes very well the measured cell degradation both for the UV and the AM0 test. The slightly higher degradation in short circuit current for the AM0 test samples compared to the theoretical curve results from contamination effects. 


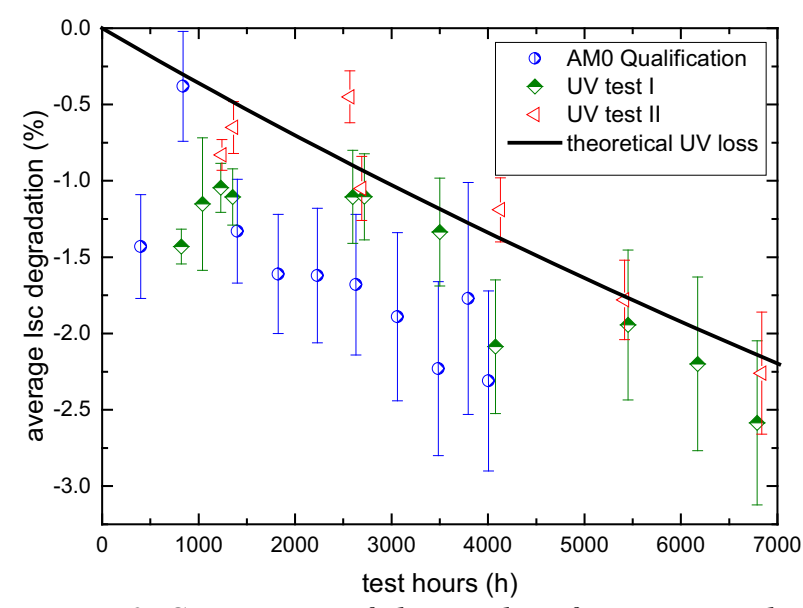

Figure 2. Comparison of electrical performance results for AMO and UV tests.

\subsection{External Quantum Efficiency}

At begin of test (BOT) and end of test (EOT), the external quantum efficiency of the SCAs has been measured. With this measurement technique, essential changes in the solar cell behaviour can be observed. Exemplarily for the SCA with the highest $I_{s c}$ degradation of $-3.5 \%$ the external quantum efficiency is shown in Fig. 3.

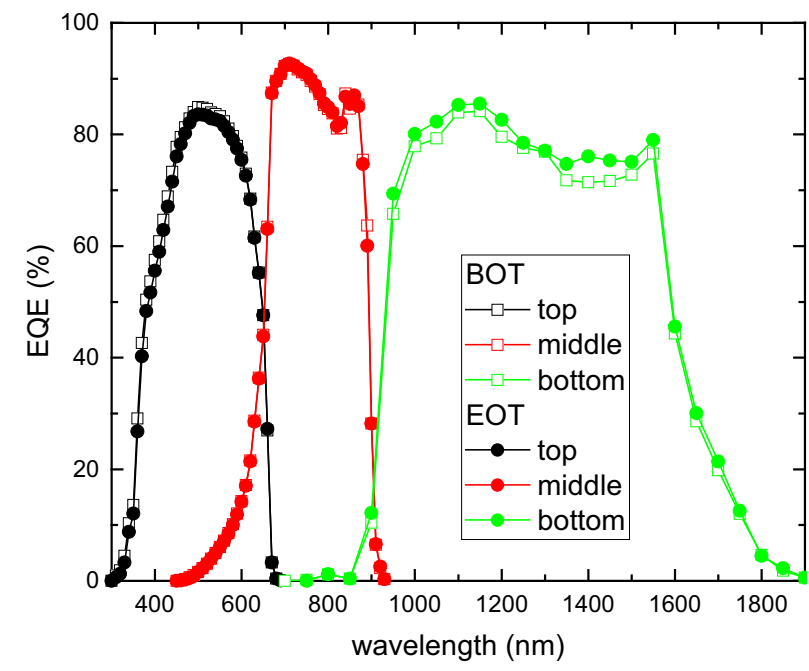

Figure 3. External quantum efficiency for the SCA with highest $I_{s c}$ degradation.

No evidence for drastic changes in the solar cell characteristics has been determined. For the same cell the ratio of the remaining factor for the EQE of the top and middle cells is shown in Fig. 4 to see more details. The bottom cell is not considered because of higher uncertainties in that measurement. Furthermore, the bottom cell will not limit the triple-junction solar cell current under any mission point and condition.

It is clearly visible that the degradation takes mainly place in the short UV wavelength range. For the first point up to $350 \mathrm{~nm}$ it has to be mentioned that the

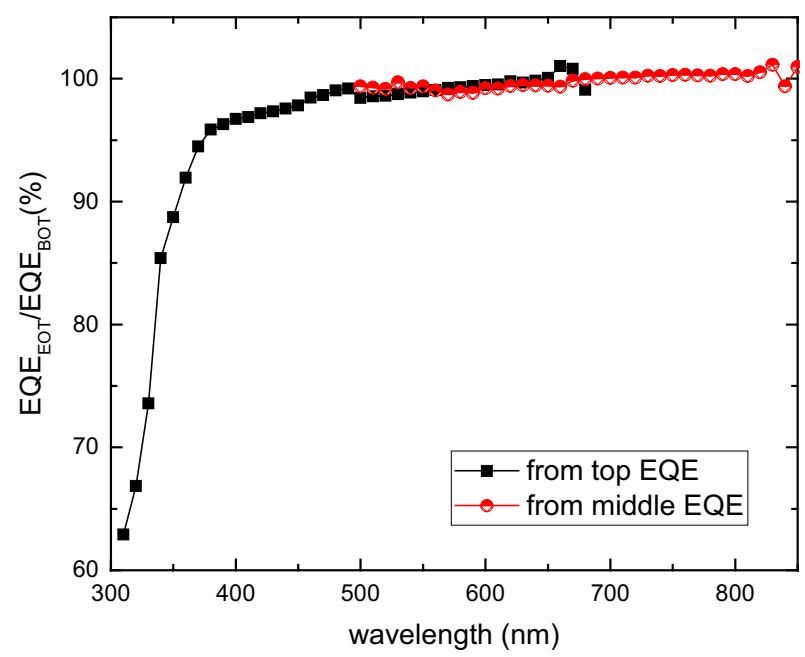

Figure 4. Ratio (remaining factor) of the EQE for the top and middle cells.

absolute values of the EQE are pretty small which leads to higher measurement uncertainties.

The EQE can be converted to spectral response (SR) values in units of $\mathrm{A} / \mathrm{W}$ and together with the AM0 sun spectrum the short circuit current $I_{s c}$ can be calculated:

$$
I_{s c}=\int S R_{t o p}(\lambda) \cdot A M 0(\lambda) d \lambda
$$

By using the top cell SR, a top cell limitation is assumed, which is correct for not particle irradiated cells. Calculating the $I_{s c}$ degradation of the cell from Fig. 3 and Fig. 4 according to Eq. (2) leads to theoretical degradation under AM0 of $-1.4 \%$. This value is lower than the measured one because of two reasons: the accuracy of the EQE measurement itself and the spectrum of the used flasher system for the electrical performance measurements is of cause not identical to the real AM0 sun spectrum.

\subsection{Thermo-Optical Measurements}

Another important topic is the change of thermo-optical properties of the solar cells with time. A degradation in absorption or emissivity could strongly influence the whole solar array performance as it would lead to higher end of life cell temperatures. The AM0 Long Duration Illumination Test was therefore used to confirm the stability of the thermo-optical properties. Only minor changes within measurement accuracy have been determined. The range of the solar absorptance $\alpha_{s}$, the IR normal thermal emittance $\varepsilon_{N}$ as well as the corresponding ratio $\alpha_{s} / \varepsilon_{N}$ is shown in Tab. 2 .

\begin{tabular}{|c|c|c|c|}
\hline & $\alpha_{s}(-)$ & $\varepsilon_{N}(-)$ & $\alpha_{s} / \varepsilon_{N}(-)$ \\
\hline Min & 0.84 & 0.82 & 0.97 \\
\hline Max & 0.90 & 0.88 & 1.04 \\
\hline
\end{tabular}

Table 2. Range of the thermo-optical parameters for the SCAs without difference for BOT and EOT. 


\section{COUPON SEGMENTS}

In addition to the single qualification samples described above, also six PVA representative coupon segments have been part of the AM0 Test. Each coupon segment has been equipped with six $40 \times 40 \mathrm{~mm}^{2}$ solar cells connected to external shunt diodes and placed next to optical surface reflectors (OSRs). A picture including all six coupon segments is depicted in Fig. 5. Due to late availability of one key component, the coupons were not tested from the very beginning and a total test duration of $3166 \mathrm{~h}$ was reached. The aim of the coupon segments was to qualify the performance of the SCAs including their surroundings, i.e. OSRs, adhesives and facesheet, which therefore covers also contamination issues.

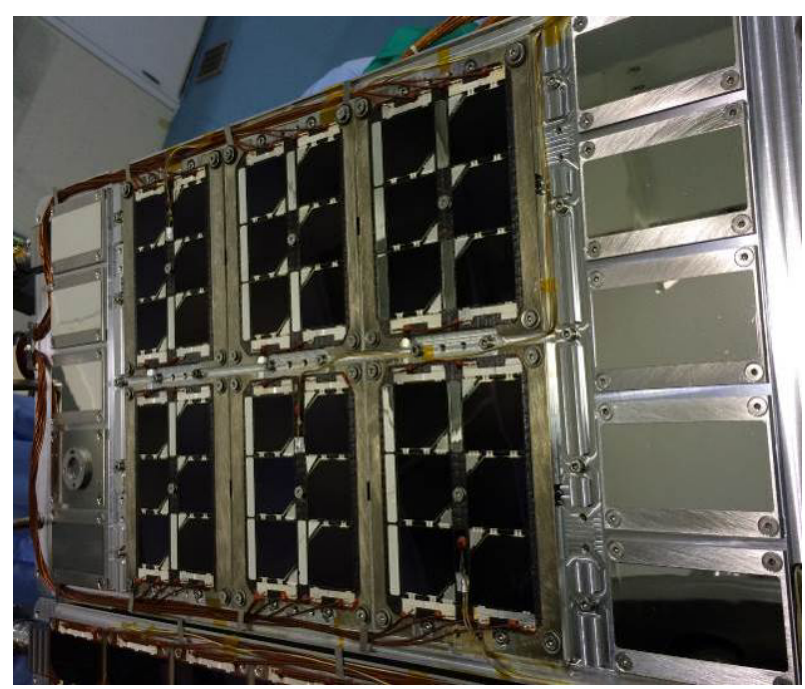

Figure 5. Picture of six coupon segments including solar cells, shunt diodes and OSRs.

The electrical characteristics of the solar cells interconnected to short strings was also measured at BOT, at intermediate inspection steps and EOT. The average degradation of all relevant electrical parameters is shown in Tab. 3 .

\begin{tabular}{|c|c|c|c|c|c|}
\hline $\begin{array}{c}\Delta P_{\max } \\
(\%)\end{array}$ & $\begin{array}{c}\Delta V_{p \max } \\
(\%)\end{array}$ & $\begin{array}{c}\Delta I_{\max } \\
(\mathbf{\%})\end{array}$ & $\begin{array}{c}\Delta I_{s c} \\
(\%)\end{array}$ & $\begin{array}{c}\Delta V_{o c} \\
(\%)\end{array}$ & $\begin{array}{c}\Delta F F \\
(\%)\end{array}$ \\
\hline-2.4 & -0.3 & -2.1 & -1.4 & -0.3 & -0.7 \\
\hline
\end{tabular}

Table 3. Average degradation of the six coupons after 3166 h test duration.

The degradation is like for the SCAs mainly for the current parameters. The lower degradation values are caused by the shorter test duration. Additionally, the configuration with strings instead of single solar cells can lead to lower degradation in short circuit current, if the limiting cell in the string has a comparatively low degradation. The overall electrical performance results of the coupon segments confirm the suitability of the whole PVA ensemble for the $\mathrm{BC}$ and $\mathrm{SolO}$ mission needs.
With this coupon segments it was not only possible to characterize the behaviour of the solar cells themselves, but also to measure the characteristics of the utilised shunt diodes. The individual shunt diodes have been measured at ambient conditions before assembly with the solar cells. After the long duration illumination test, the shunt diodes were electrically separated from the solar cells by cutting the interconnectors. The measurement of the EOT shunt diode characteristics was performed by using a contact technique as shown in Fig. 6.

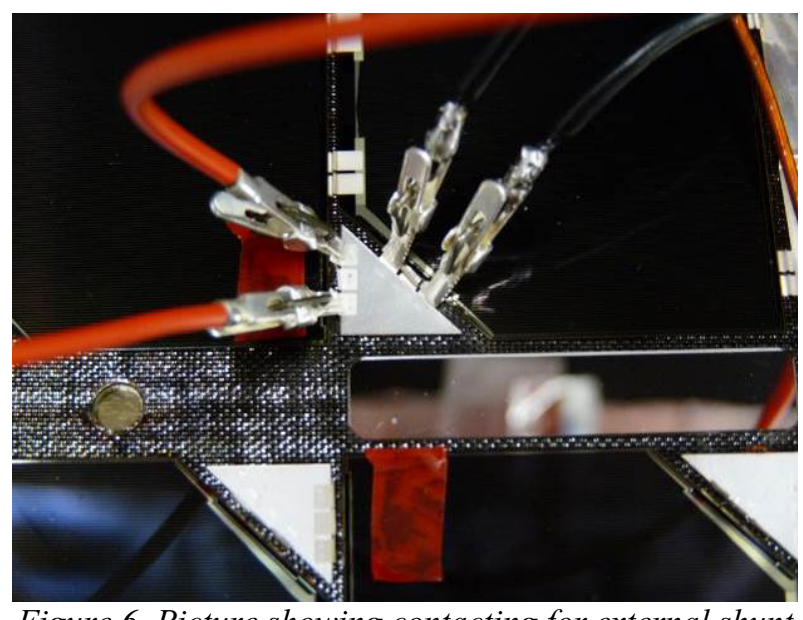

Figure 6. Picture showing contacting for external shunt diode characterization.

The average results for BOT and EOT are shown in Tab. 4.

\begin{tabular}{|c|c|c|}
\hline & $V_{\text {forw }}(@ 3 \mathrm{~A})(\mathrm{V})$ & $I_{\text {rev }}(@-4 \mathrm{~V})(\mu \mathrm{A})$ \\
\hline BOT & 0.842 & 0.004 \\
\hline EOT & 0.805 & 0.070 \\
\hline
\end{tabular}

Table 4. Average shunt diode parameters BOT and EOT at ambient conditions.

The forward voltage at a current level of $3 \mathrm{~A}$ was slightly reduced by about $40 \mathrm{mV}$ over the test duration. This is even an improvement/advantage, because the voltage loss is reduced in case of a forward biased shunt diode. The reverse current at $-4 \mathrm{~V}$ was slightly increased, but is in average and also for each individual diode still well below the BOT acceptance criteria of $<2$ $\mu \mathrm{A}$.

\section{CHALLENGES DURING TEST EXECUTION}

The setup for this very specific test was new developed. Besides expected measures to compensate the lamp degradation, also some unexpected challenges occurred during test execution. Some examples are explained in more detail below.

\subsection{Lamp movement and degradation}

The absolute beam intensity at the location of the 
sample plates was determined with a lamp mapping in three planes BOT and EOT for each test cycle. The 25 $\mathrm{kW}$ Xenon lamps used showed on the one hand an unpredictable movement of the beam as no optics was present in the beam, which resulted in intensity resp. temperature variations. This was compensated as good as possible by applying an appropriate thermal washer configuration below the samples [4]. On the other hand, the lamp intensity suffered from certain intensity degradation with minor differences between the individual lamps. This was taken into account by a stepwise increase of lamp current. To decide on the point in time and the amount of lamp current increase, the temperature readings of the IR camera and the thermocouples on the witness cells as well as the individual cell currents from the live data acquisition were used. The average solar cell current values for all samples on one plate over $24 \mathrm{~h}$ were used for this evaluation. By doing this, the movement of the lamp, which has also influence on the individual cell current, was excluded as much as possible.

\subsection{Test Artefact}

As described above, an IR camera inside the chamber was used to monitor the arising solar cell temperatures. After starting the first exposure cycle, so called "red corners" have been observed for the large qualification samples only (see Fig. 7). This could be explained by a edge delamination of the solar cell from the aluminium holder plate induced by the different coefficient of thermal expansion (CTE) of both materials. As the shear stress in the bond line for the large cells is twice as big as for the small cells, this phenomenon was reduced to the $80 \times 40 \mathrm{~mm}^{2}$ qualification samples. It could be proven both by analysis and test that this was a test artefact only. This phenomenon is observed at high intensities/temperatures and additionally under vacuum conditions as the weaker thermal contact between cell and holder plate causes a bad temperature distribution. In the flight hardware configuration with panel substrates out of carbon fibre instead of aluminium plates for the test sample holders, the CTE difference is much smaller and this effect cannot occur.

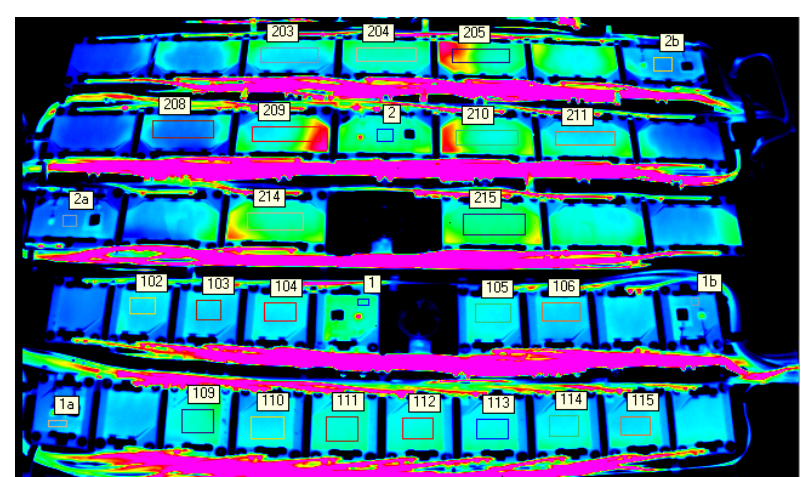

Figure 7. IR picture of BepiColombo qualification samples.

\subsection{Wire Darkening}

During the exposure cycles, the qualification samples had to be tested under operating conditions. Therefore, cabling of the individual samples had to be present which led to unrepresentative high cable bundles exposed to the illumination. This setup is in contrast to the solar array flight configuration as the cables are routed on the panel rear side. Due to the height, there was a bad thermal contact of the cable bundles to the aluminium sample plates. The wires therefore have been exposed to extremely high temperatures which caused darkening of the wires and also certain contamination. To allow continuation of the test, cable shields have been introduced to protect the cabling from further degradation. These aluminium shields are shown in Fig. 8 .

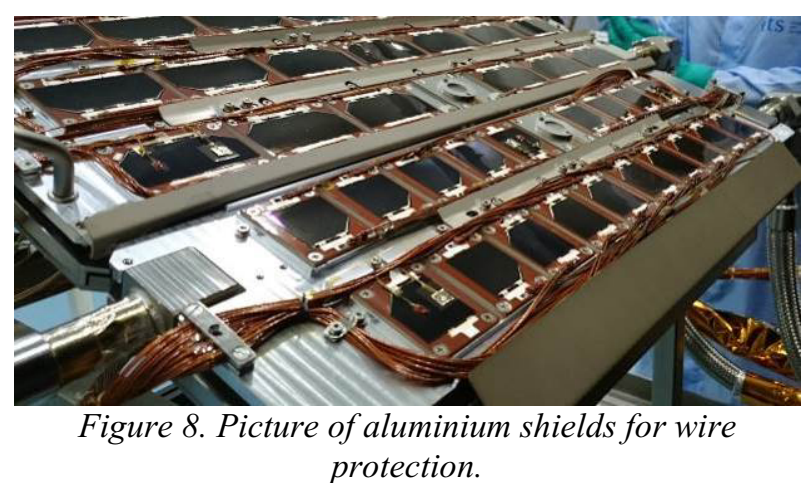

\section{FIRST SOLAR ORBITER QUALIFICATION TEST RESULTS}

The SolO representative $16 \quad 40 \times 40 \quad \mathrm{~mm}^{2}$ single qualification cells have been tested further in the SolO AM0 Qualification Test for 1000 hours under the required higher intensity of up to $12.8 \mathrm{SCs}$ leading to an overall test duration of 5000 hours for these samples. This additional test with $1000 \mathrm{~h}$ was split in three test runs. To optimize the available test setup, the qualification samples were re-arranged to center them in the region with the highest illumination homogeneity. These new sample locations are depicted in green in Fig. 9 (a). The other positions were equipped with dummy cells to have the same thermo-optical properties like in the BepiColombo test. Therefore, it was not necessary to change the thermal washer concept compared to the previous test [4]. A picture from the test samples on the MGSE is shown in Fig. 9 (b).

The main focus of the characterization was again the electrical performance measurement of the individual SCAs after each test cycle. Prior to the SolO test start a new BOT measurement was performed, with slightly higher values compared to EOT from the BC AM0 test. The difference is within the measurement accuracy, but could be an indication for a small annealing during the storage period between the two qualification tests. The average degradation of the 16 present SCAs in the SolO 

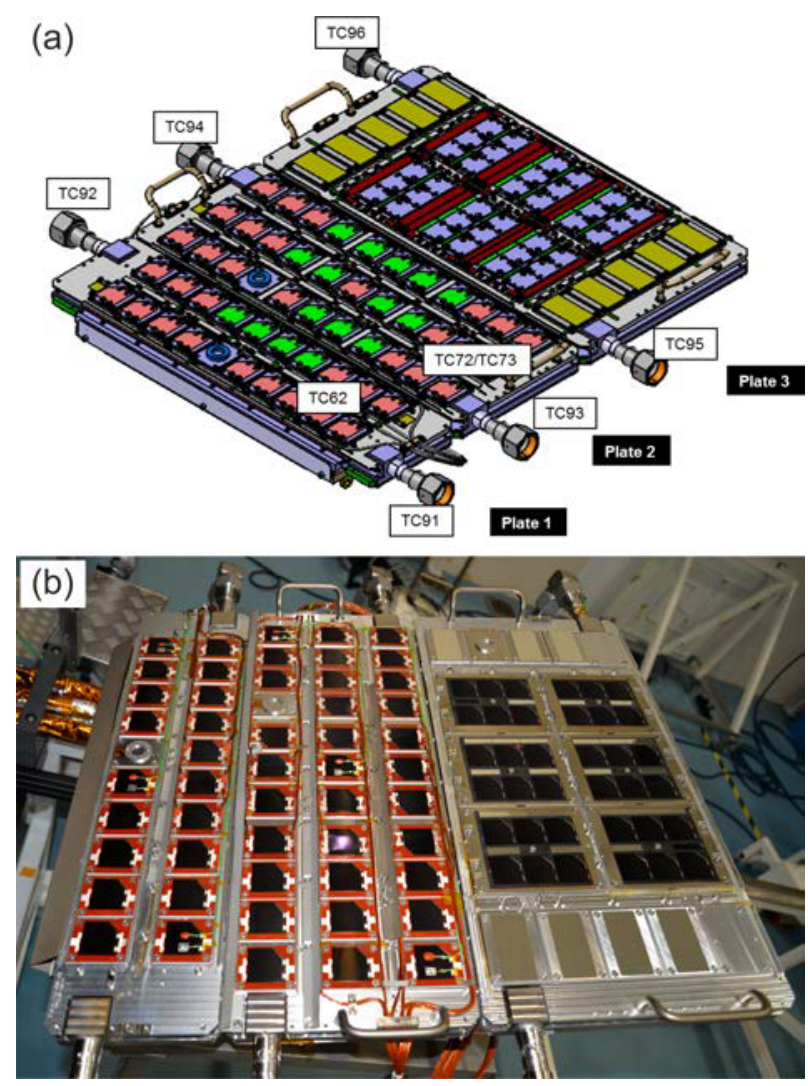

Figure 9. (a) Overview of sample locations for SolO Qualification Test (shown in green). (b) Picture of the samples on the MGSE.

Qualification Test compared to BOT SolO (1000 h) and BOT BC (5000 h) are listed in Tab. 5.

\begin{tabular}{|c|c|c|c|c|c|c|}
\hline$\Delta$ to & $\begin{array}{c}\Delta P_{\max } \\
(\%)\end{array}$ & $\begin{array}{c}\Delta V_{\max } \\
(\%)\end{array}$ & $\begin{array}{c}\Delta I_{p \max } \\
(\%)\end{array}$ & $\begin{array}{c}\Delta I_{s c} \\
(\%)\end{array}$ & $\begin{array}{c}\Delta V_{o c} \\
(\%)\end{array}$ & $\begin{array}{c}\Delta F F \\
(\%)\end{array}$ \\
\hline $\begin{array}{c}\text { BOT } \\
\text { SolO }\end{array}$ & -0.8 & -0.5 & -0.3 & -0.3 & -0.4 & -0.1 \\
\hline $\begin{array}{c}\text { BOT } \\
\text { BC }\end{array}$ & -3.1 & -0.5 & -2.6 & -2.5 & -0.5 & -0.1 \\
\hline
\end{tabular}

Table 5. Average degradation of SolO qualification $S C A s$ for the additional $1000 \mathrm{~h}$ test duration and compared to BOT BC with 5000 h in summary.

For this additional long duration illumination test with $1000 \mathrm{~h}$ only a small additional degradation in the current parameters was observed, which is a consistent continuation of the BC AM0 test. The variations in the voltage parameters are mainly caused by measurement accuracy. This can be seen in the $V_{p \max }$ parameter, which was in average identical for BOT BC and BOT SolO. The updated graph from Fig. 2 with the results from the SolO qualification test is shown in Fig. 10.

The offset between the theoretical UV degradation and the real measurements is relatively stable after short test duration. This means the root cause for the higher degradation, which is most likely contamination, occurs mainly at the beginning and saturates later. This is an

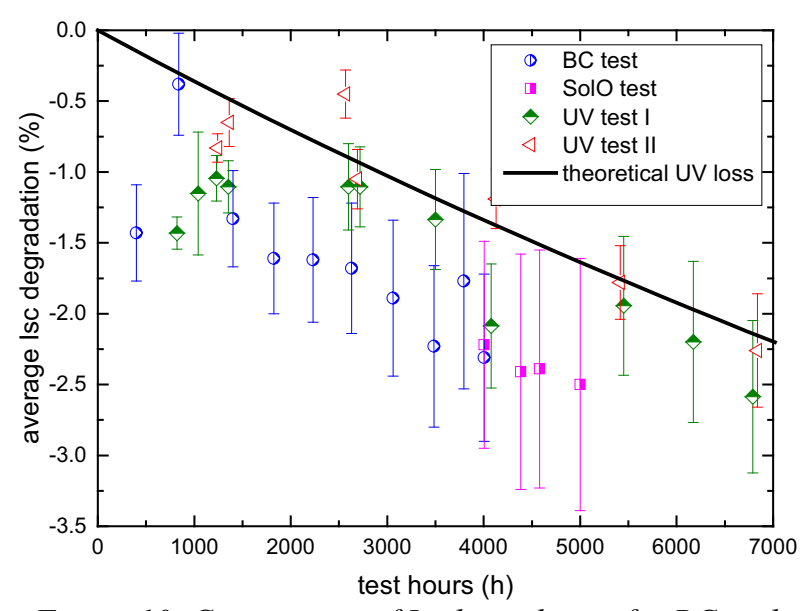

Figure 10. Comparison of $I_{s c}$ degradation for $B C$ and SolO AMO and UV tests.

expected behaviour for the outgassing of adhesive, for example.

\section{CONCLUSIONS}

The BepiColombo and Solar Orbiter AM0 Long Duration Illumination Qualification Tests have been successfully completed. The validity of the solar cell design for these high temperature, high intensity missions needs could be confirmed. The results with respect to solar cell behaviour and further characteristics have been presented. In addition, some insights have been given in challenges which occurred during test execution.

\section{ACKNOWLEDGMENTS}

The work on the BepiColombo and Solar Orbiter solar array including this tests was carried out under a programme of and funded by the European Space Agency. The view expressed herein can in no way be taken to reflect the official opinion of the European Space Agency.

\section{REFERENCES}

1. Zimmermann, C.G., Nömayr, C., Köstler, W., Caon, A., Fernandez E., Baur, C. \& Fiebrich H.K. (2011). Photovoltaic Technology Development for the BepiColombo Mission. In Proc. $9^{\text {th }}$ European Space Power Conference ESPC 2011, 2103064.

2. Zimmermann, C.G., Nömayr, C., Kolb, M. \& Caon, A. (2011). A solar cell design for the BepiColombo high intensity - high temperature mission. In Proc. IEEE Photovoltaic Specialists Conference PVSC 2011, pp. 003713-003718.

3. Andreev, T., Zimmermann, C., Löhberg, A., de Jong, S., Schuhmacher, U., Schneider, J., Köstler, W., Parketta, P., Zäh, E., Holzbauer, R., Fink, M., Hall, J., Fernandez Lisbona, E., Lundmark, K., 
Baur, C., Taylor, S., Panin, F., Caon, A. (2015). Qualifying Solar Array Components for High Intensity, High Temperature Environments - The Test Approach for the Bepi Colombo Solar Arrays, In Proc. 10th European Space Power Conference ESPC 2014, ESA SP-719 (CD-ROM).

4. Oberhüttinger, C., Quabis, D. \& Zimmermann, C.G. (2014). Simulating large area, high intensity AM0 illumination on earth - representative testing at elevated temperatures for the BepiColombo and SolO missions. In Proc. 10th European Space Power Conference ESPC 2014, ESA SP-719 (CDROM).

5. Zimmermann, C.G. (2008). Time dependent degradation of photovoltaic modules by ultraviolet light. Appl. Phys. Lett. 92, 241110. 